\title{
Tratamento cardiorrespiratório de pacientes com Síndrome Pós-Poliomielite
}

\author{
Cardiorespiratory treatment of patients with \\ Post-Polio Syndrome
}

\author{
Marco Orsini ${ }^{1}$, Mauricio de Sant'Anna Junior ${ }^{2}$, Carlos Eduardo \\ Cardoso $^{3}$, Rossano Fiorelli ${ }^{3}$; Stenio Fiorelli ${ }^{3}$; Gisele Roque de Souza ${ }^{3}$; \\ Eduardo Jorge C. da Silva ${ }^{4}$, Adalgiza Mafra Moreno ${ }^{5}$, Acary S. B. \\ Oliveira $^{6}$; Adriana Leico Oda ${ }^{6}$; Marcos RG de Freitas ${ }^{7}$; Victor Hugo \\ Bastos $^{8}$; Silmar Teixeira ${ }^{8}$
}

1. Universidade de Vassouras - Programa de Mestrado em Ciências Aplicadas em Saúde, Universidade Iguaçu - UNIG, Laboratório de Mapeamento Cerebral e EEG, Rio de Janeiro-RJ, Brasil.

2.Instituto Federal de Educação, Ciência e Tecnologia do Rio de Janeiro, Rio de Janeiro-RJ, Brasil.

3. Universidade de Vassouras-RJ, Brasil. Mestrado em Ciências Aplicadas em Saúde e Faculdade de Medicina.

4. Hospital Pedro Ernesto, UERJ, Departamento de Neurofisiologia. Rio de Janeiro-RJ, Brasil.

5.Universidade Iguaçu - UNIG, Brasil.

6.Universidade Federal de São Paulo, Departamento de Neurologia, São Paulo-SP, Brasil.

7. Universidade Federal do Rio de Janeiro - Departamento de Neurologia - RJ, Brasil.

8. Universidade Federal do Piaui - Departamento de Fisioterapia - UFPI, Brasil.

\section{Resumo}

Objetivo. Síndrome pós-polio (SPP) é uma condição que afeta sobreviventes da poliomielite aguda, anos após a recuperação de um ataque agudo inicial do vírus. $\mathrm{Na}$ maioria das vezes, os sobreviventes da polio começam a apresentar nova paresia gradual nos músculos que foram previamente afetados pela infecção. A incidência real de doenças cardiovasculares em indivíduos que sofrem de SPP não é conhecida. Entretanto, há indícios para suspeitar de que sujeitos com SPP podem estar em maior risco. Método. Realizou-se uma busca de artigos nas bases de dados: Bireme, Scielo e Pubmed, utilizando as seguintes palavras-chave: síndrome pós-poliomielite, função cardiorrespiratória e reabilitação, nos idiomas Inglês, Francês e Espanhol. Embora tenhamos selecionado um número expressivo de artigos, somente foram considerados os duplo-cegos, randomizados-controlados, além de consensos. Resultados. Certas características da SPP, tais como fadiga muscular, paresia, dor muscular e/ou articulares podem resultar em descondicionamento por inatividade física, além de obesidade e dislipidemia. Dificuldades respiratórias são comuns e podem resultar em hipoxemia. Conclusão. Somente quando avaliados e tratados em tempo hábil, alguns pacientes são capazes de obter os benefícios do uso dos músculos respiratórios auxiliares em termos de qualidade de vida.

Unitermos. síndrome pós-poliomielite; transtornos respiratórios; reabilitação

\begin{abstract}
Objective. Post-polio syndrome (PPS) is a condition that affects polio survivors years after recovery from an initial acute attack of the poliomyelitis virus. Most often, polio survivors experience a gradual new weakening in muscles that were previously affected by the polio infection. The actual incidence of cardiovascular diseases in individuals suffering from PPS is not known. However, there is a reason to suspect that individuals with PPS might be at increased risk. Method. A search for papers was made in the databases Bireme, Scielo and Pubmed with the following keywords: post-polio syndrome, cardiorespiratory and rehabilitation in English, French and Spanish languages. Although we targeted only seek current
\end{abstract}


studies on the topic in question, only the relevant (double-blind, randomizedcontrolled and consensus articles) were considered. Results. Certain features of PPS such as generalized fatigue, generalized and specific muscle weakness, joint and/or muscle pain may result in physical inactivity deconditioning obesity and dyslipidemia. Respiratory difficulties are common and may result in hypoxemia. Conclusion. Only when evaluated and treated promptly, some patients can obtain the full benefits of the use of respiratory muscles aids as far as quality of life is concerned.

Keywords. post-poliomyelitis syndrome; respiration disorders; rehabilitation

Trabalho realizado na Universidade Federal do Rio de Janeiro, rio de Janeiro-RJ, Brasil.

Conflito de interesse: não

Recebido em: 2018

Aceito em: 2018

Endereço para correspondência: Marco Orsini. Rua Tavares de Macedo, 95/902. Icaraí. Niterói-RJ. CEP: 24220-215. E-mail: orsinimarco@gmail.com

\section{INTRODUÇÃO}

As doenças neuromusculares (DNDs) podem afetar qualquer pessoa a qualquer momento. Elas causam fraqueza muscular que pode afetar os sistemas cardiovascular, respiratório, além da tosse, deglutição, fala e os músculos do esqueleto apendicular, causando incapacidades e comprometimento da qualidade de vida. A poliomielite aguda anterior (PAA) pode ser definida como uma doença humana endêmica causada por um enterovírus de distribuição mundial, que compromete as células do corno anterior da medula espinhal ${ }^{1,2}$. Um novo quadro para a amiotrofia, fadiga incomum, problemas articulares e dor, todos com uma característica progressiva, marca a síndrome pós-pólio (SPP).

A prevalência de fadiga é notavelmente alta e pode contribuir significativamente para a incapacidade dos pacientes e uma qualidade de vida reduzida. Outras apresentações clínicas possíveis são a intolerância ao frio e 
problemas cardiorrespiratórios, incluindo a apneia do sono. Ressaltamos que, embora exista uma ampla gama de sinais e sintomas, sua totalidade não se expressa em todos os indivíduos acometidos ${ }^{1,2}$.

Não encontra-se disponível um teste eletrodiagnóstico específico para o PPS. A eletromiografia convencional é incapaz de distinguir confiavelmente pacientes com póspólio estáveis e aqueles com nova fraqueza. Entretanto, a avaliação clínica e eletrofisiológica pode ser útil para documentar sinais de perda prévia do neurônio motor devido à PAP e para excluir outros distúrbios neurológicos. O diagnóstico do PPS é apoiado por novos achados clínicos e evidências clínicas e eletrofisiológicas da $\mathrm{PAA}^{3}$.

Pacientes com PAA podem ter comprometimento da função pulmonar, independentemente do relato de dispneia. Fatores de risco, como ventilação no início da polio e início da poliomielite após dez anos de idade, podem ajudar a predizer aqueles com risco de comprometimento respiratório. A medida da força muscular respiratória e da pressão expiratória máxima $\left(P E_{\text {máx }}\right)$ em particular, podem representar um maior risco para o declínio da função pulmonar nessa população ${ }^{4}$.

\section{MÉTODO}

Realizou-se uma busca de artigos nas bases de dados: Bireme, Scielo e Pubmed, utilizando as seguintes palavraschave: síndrome pós-poliomielite, função 
cardiorrespiratória e reabilitação, nos idiomas Inglês, Francês e Espanhol. Embora tenhamos selecionado um número expressivo de artigos, somente foram considerados os duplo-cegos, randomizados-controlados, além de consensos.

\section{RESULTADOS E DISCUSSÃO \\ Distúrbios do sono}

Os distúrbios do sono são comuns em pacientes com $\mathrm{SPP}^{5}$. Sua natureza insidiosa pode dificultar 0 reconhecimento desses distúrbios por pacientes, familiares e cuidadores ${ }^{6}$. Um estudo com registro de nove meses de seguimento de 21 pacientes com SPP não foi capaz de evidenciar diferenças significativas de qualidade do sono entre portadores de SPP, não portadores e grupos controle ${ }^{7}$.

Entre os distúrbios do sono relacionados à SPP, o movimento periódico dos membros e, principalmente, apneia obstrutiva do sono (OSA) são as mais frequentes $^{3,6,8}$. Os distúrbios respiratórios parecem ser mais comuns durante o sono nos pacientes com SPP do que na população geral $^{8}$. Existem alguns sinais e sintomas que podem alertar para uma possível ventilação ineficiente, predispondo a insuficiência respiratória, tais como: ortopneia, insônia, ansiedade ao ir dormir, sono fragmentado, dores de cabeça matinais e piora do estado mental, dificuldade de concentração ${ }^{6}$. 
Um estudo prospectivo com 60 pacientes com SPP mostrou que alterações do sono estavam presentes em $78,3 \%$ dos pacientes. A eficiência do sono diminuiu em 44 dos 60 pacientes, com $67,4 \%$ dos pacientes apresentando AOS e utilizando ventilação não invasiva. Esta ineficiência é atribuída a mais de 10 despertares por hora. O exame polissonográfico demonstrou maior tempo gasto no sono superficial do que em estágios mais profundos ${ }^{8}$. Essas alterações do sono podem ser creditadas à deficiência induzida pelo poliovírus na formação reticular, uma área conhecida por mediar início do sono REM ${ }^{8,9}$.

Em caso de AOS, a utilização de BI-level Positive Airway Pressure (BiPAP) a realização de fisioterapia respiratória, bem como a vacinação contra gripe estão entre os recursos são indicados ${ }^{6,8,10}$. Estes distúrbios do sono podem contribuir para um sintoma cardinal da SPP: fadiga ${ }^{5,8,11,12}$. Considerando sua importância, algumas abordagens tentaram minimizar seu impacto. Modafenil não se mostrou superior ao placebo para alivio da fadiga ou melhorar a qualidade de vida em amostra de 36 pacientes com SPP. Entretanto, um ensaio controlado demonstrou que a estimulação transcraniana por corrente contínua (ETCC) sobre as áreas pré-motoras por quinze dias melhorou o sono e sintomas de fadiga em pacientes com SPP ${ }^{13}$.

A fadiga generalizada, fraqueza muscular específica, dores articulares e/ou musculares podem resultar em inatividade, descondicionamento, obesidade e dislipidemia. PAA e SPP com dificuldades respiratórias podem 
desenvolver hipoxemia. Qualquer uma destas circunstâncias (descondicionamento, obesidade, dislipidemia e hipoxemia) pode aumentar o risco de doenças cardiovasculares doença ${ }^{14}$. A segurança e a eficácia da prática de exercício em pacientes com SPP e outras DNMs foram investigadas por alguns pesquisadores. Todos descreveram que um programa de exercícios cuidadosamente projetado, e que evite a fadiga muscular é capaz de fornecer resultados positivos. Em geral, o exercício deve ser iniciado gradualmente e em um nível mais baixo, sempre com prescrições individualizadas com as adaptações necessárias para cada sujeito. Toda atenção deve ser voltada para os cuidados com o uso excessivo, além de aconselhamento nutricional ${ }^{15,16}$.

\section{Função pulmonar e cuidados respiratórios na síndrome pós-pólio}

A função pulmonar relacionada aos efeitos da idade de início da doença foi exaustivamente investigada em pacientes com SPP ${ }^{17,18}$. Muitos testes e técnicas diferentes provaram ser úteis a este respeito, mas ainda não existe um consenso sobre a melhor forma de usar esses testes. Todo esse esforço pode ser explicado em função da dificuldade de diagnosticar insuficiência respiratória do SPP, principalmente quando não existe suspeita ${ }^{17,19}$.

Curiosamente, em pacientes com PPS, a função pulmonar prejudicada está associada à idade de início (progressão de dez anos ou mais), a necessidade de 
ventilação mecânica durante a fase aguda da doença, quadriparesia e tempo de exposição à doença ${ }^{17}$. Em outro estudo, foi observada uma associação entre disfunção respiratória e a presença de cifoescoliose ou paresia diafragmática ${ }^{20}$.

Geralmente, a PPS afeta os mesmos grupos musculares afetados no início da PAA. Assim, se a insuficiência respiratória não foi uma característica na primeira apresentação da poliomielite, é relativamente improvável que se manifeste durante na PPS. No entanto, a distribuição e gravidade do envolvimento dos músculo respiratórios em pacientes com SPP é bastante variável ${ }^{19}$. Devido à distribuição variável da paresia, existem alguns pacientes cujo a fraqueza dos músculos inspiratórios e expiratórios são aproximadamente iguais. Em outros, pode predominar fraqueza muscular expiratória ou fraqueza muscular inspiratória preferencialmente. Existe uma tendência para que a fraqueza expiratória exceda a fraqueza inspiratória ${ }^{19,21}$.

A principal manifestação respiratória da SPP na prova de função pulmonar é distúrbio restritivo. Isso ocorre devido à fraqueza dos músculos respiratórios, que são incapazes para gerar o vácuo intratorácico necessário para inspiração ou a pressão necessária para a expiração completa. Consequentemente, isso leva a reduções na capacidade pulmonar total (CPT), capacidade vital (CV) e ventilação voluntária máxima (VVM). Além disso, a VVM foi aproximadamente $27 \%$ menor em pacientes com SPP em 
comparação controles saudáveis $(p=0,0127)^{22}$. No mesmo estudo, no entanto, os autores observaram que os valores de VMM estavam dentro da normalidade quando comparado com os valores previstos para a população brasileira. Semelhante a outras doenças neuromusculares, o CV é menor quando comparada ao previsto em função da fraqueza muscular isolada na SPP. O volume residual é aumentado em alguns casos, não por obstrução das vias aéreas ${ }^{23}$.

A redução relativa da capacidade inspiratória e volume de reserva expiratória pode fornecer informações sobre 0 envolvimento relativo de músculos inspiratórios versus expiratórios, respectivamente. Uma aplicação da espirometria que pode fornecer informações úteis é comparar VC na posição sentada e supina em pacientes com SPP com suspeita de fraqueza do diafragma; um declínio de $25 \%$ ou mais quando os pacientes assumem a posição supina é considerado um indicador de fraqueza significativa ${ }^{23}$.

A redução da complacência pulmonar, assim como a redução da expansibilidade da parede torácica é outra possível complicação do possível na SPP. A redução na complacência pulmonar associada à redução dos volumes pulmonares isso é característico da doença neuromuscular. Essas alterações podem causar microatelectasia, redução da tensão superficial, alterações nas fibras elásticas do interstício pulmonar, ou uma combinação de um ou mais 
desses fatores, cada um que pode resultar de baixos volumes pulmonares habitualmente ${ }^{23}$.

Outras complicações tardias que podem estar relacionadas à SPP são hipoventilação alveolar crônica, alterações no controle central da respiração e síndrome da apneia do sono. Estudos envolvendo a investigação de pressões respiratórias estáticas máximas confirmam que a hipercapnia só se desenvolve quando a força muscular respiratória é consideravelmente reduzida.

O comprometimento do sistema respiratório superior também pode ser um fator importante, mas facilmente negligenciado, no PPS ${ }^{24}$. Alguns pacientes com SPP tendem a respirar com uma padrão que compromete sua função ventilatória aumentando a relação entre espaço morto e capacidade vital. Quando a carga ventilatória no sistema respiratório é excessiva a capacidade ventilatória, os pacientes desenvolvem falta de ar, tolerância ao exercício reduzida e, eventualmente, insuficiência respiratória ${ }^{24}$. Além disso, a insuficiência respiratória é o fator limitante para o exercício em pacientes com SPP com disfunção muscular respiratória ${ }^{18}$. Segundo esses autores, a extensão da doença pulmonar é evidente através de pelos valores de gases sanguíneos em situações de esforço, mas não em repouso ${ }^{18}$.

A abordagem do cuidado respiratório em pacientes com SPP segue as recomendações padrão para pacientes com doenças neuromusculares, com poucas particularidades. Como a fraqueza muscular expiratória é 
uma manifestação comum da doença, a tosse assistida manualmente ou mecanicamente pode ser útil em pacientes selecionados com o objetivo de prevenir a retenção de secreções ${ }^{25}$.

Além disso, quando os músculos inspiratórios são significativamente comprometidos, outros recursos como técnica de empilhamento da respiração, hiperinsuflação manual (com bolsa ressuscitadora) e respiração com pressão positiva intermitente (RPPI) com utilização de ventiladores de pressão positiva aplicados de forma não invasiva por um bocal ou máscara, também podem ser usados para melhorar o volume inspiratório antes da manobra expiratória forçada ${ }^{26}$.

Em pacientes gravemente comprometidos, tanto para a fase inspiratórios quanto para a fase expiratória pode ser utilizada a tosse mecanicamente assistida, através de um dispositivo especialmente concebido para melhorar a tosse em doenças neuromusculares. Pacientes com pico da tosse inferior à 270L/min devem ser considerados elegíveis para técnicas de aumento do fluxo de pico da tosse ${ }^{27}$. Da mesma forma, as técnicas de expansão pulmonar também são utilizadas para manter ou melhorar a complacência pulmonar e a tosse, além de diminuir a existência de atelectasia $^{28}$.

Alguns pacientes podem se beneficiar da utilização da ventilação não invasiva (VNI) Notavelmente, aqueles com sinais e sintomas clínicos de fracasso e hipoventilação são os que mais se beneficiam, além disso, pacientes com 
alguns sintomas, como dispneia aos pequenos esforços ou fala, ortopneia, despertares noturnos frequentes, sonolência diurna, fadiga diurna, cefaléia matinal, dificuldade em eliminar secreções, apatia, falta de apetite, falta de concentração ou memória ${ }^{29}$. A utilização da VNI geralmente começa com o uso noturno e durante o dia, conforme necessário. Quando o paciente cursa com vários episódios de dessaturações (abaixo de 95\%) e o $\mathrm{CO}_{2}$ encontra-se superior a $50 \mathrm{mmHg}$, um teste com utilização de VNI noturna pode ajudar na redução de sintomas e também a normalizar gases sanguíneos ${ }^{28}$.

Quase metade dos pacientes com SPP apresentam algum distúrbio do sono, especificamente fadiga e sonolência diurna, dores de cabeça e síndrome das pernas inquietas ${ }^{30}$. Os principais achados clínicos são hipoventilação, apneia e hipopneia associada a obstruções significativas, dessaturação, redução da eficiência do sono ${ }^{8,31}$. Recomenda-se que pacientes com suspeita de disfunção do sono decorrentes de doenças neuromusculares sejam investigados com polissonografia de noite inteira em laboratório $^{32}$. Esta ferramenta é valiosa para o diagnóstico e adequação do tratamento, uma vez que permite a titulação pressórica para utilização da $\mathrm{VNI}^{33}$.

\section{Problemas cardiológicos e reabilitação na síndrome pós-pólio}

As doenças cardiovasculares têm alta incidência e prevalência, progredindo para desfechos de morbidade e 
mortalidade na população em geral, e não devem ser ignoradas nos pacientes com SPP. A exposição frequente a fatores de risco para doenças cardiovasculares, particularmente para doença arterial coronariana (DAC), como história familiar de DAC, obesidade, diabetes mellitus, tabagismo, hipertensão, aumento do colesterol LDL, redução do colesterol HDL e dislipidemia podem aumentar o risco de DAC em pacientes com SPP em virtude dos baixos níveis de atividade física e até mesmo sedentarísmo ${ }^{14,34}$.

A adequada estratificação do risco cardiovascular para pacientes com SPP pode levar em consideração o uso de diversos questionários (Framingham, Münster Heart Study, Reynolds etc.). Contudo, o uso de escore de cálcio coronariano (ECC) pode ser uma excelente alternativa devido ao seu bom poder de previsão para eventos coronarianos, já evidenciado em várias análises multivariadas e atualmente descrito como uma ferramenta importante para a estratificação do risco cardiovascular, por detecção de aterosclerose subclínica. Como não requer contraste intravenoso, detecção do ECC não requer jejum ou qualquer outro tipo de preparação ${ }^{35}$.

Outra questão relevante para avaliação do sistema cardiovascular em portadores de SPP é a realização de ecocardiograma e eletrocardiograma para avaliação da função do ventrículo esquerdo e condução elétrica, respectivamente. Um estudo observacional e prospectivo mostrou um aumento na chance de pacientes com doenças neuromusculares evoluírem com hipertrabeculação e/ou 
não compactação de ventriculo esquerdo, sendo os pacientes com SPP incluídos neste grupo ${ }^{36}$. Tal condição pode resultar em arritmias complexas, além de predispor a morte súbita, em grande parte dos casos essa condição é assintomática. Acredita-se que para a redução do risco cardiovascular fatores nos sujeitos com SPP, é necessário a orientação sobre a mudança de hábitos, como a educação nutricional. No PPS a fator de risco variável e predominante para um aumento

A chance de desenvolver DAC ocorre em decorrencia de um estilo de vida sedentário. A fadiga e as alterações do sistema músculo-esquelético na SPP são fatores que predispõe a inatividade física resultando em descondicionamento, ganho de peso, alterações no colesterol em dislipidemia ${ }^{37,38}$. Além disso, houve redução significativa na capacidade aeróbia avaliada por ergoespirometria em $\operatorname{PPS}^{37}$. Essa redução causa limitação na realização de atividades em intensidade submáxima e máxima resultando em deterioração da capacidade funcional, por esse motivo é indicada a prática regular de exercício físico, que devem ser cuidadosamente prescritos.

No entanto, houve uma melhora significativa em indivíduos com SPP que participaram de um programa de exercício de 16 semanas, com frequência de três vezes por semana, com duração de 20 minutos em cada sessão com utilização de cicloergômetro de membro superior ${ }^{38}$. A intensidade da atividade foi definida utilizando a frequência cardíaca de reserva (70-75\%) usando a fórmula de 
Karvonen e a escala de sensação subjetiva de esforço descrita por Borg na intensidade de 13-15 pontos.

Posteriormente todos os sujeitos foram submetidos à avaliação ergoespirométrica antes e depois do programa de exercícios. Ao final houve um aumento no tempo de exercício realizado pelo grupo que praticou a atividade, além de um aumento $\mathrm{VO}_{2 \text { picor }} \mathrm{VCO}_{2}$ e $\mathrm{VE}$, além de segurança hemodinâmica durante as sessões de treinamento, promovendo benefícios em comparação ao grupo de portadores de SPP que não praticaram exercício.

Além disso, um programa de exercícios com duração de seis semanas, com intensidade de 55-70\% frequência cardíaca máxima, realizado três vezes por semana, pode desempenhar um papel significativo melhorara da resistência dos pacientes com SPP, reduzindo a fadiga durante o desempenho das atividades da vida diária ${ }^{39}$. Visando a correta prescrição da intensidade do exercício, o limiar anaeróbico pode ser obtido em indivíduos com SPP seguindo como sendo o padrão ouro para tal demanda ${ }^{40}$. No entanto, o ergômetro utilizado deve ser 0 cicloergômetro. Em caso de impossibilidade de se obter o limiar anaeróbico (ausência de ergoespirometria) o uso da frequência e da escala de Borg parecem ser a melhor alternativa $^{40}$.

A fim de verificar o risco cardiovascular em indivíduos com SPP, sugere-se o monitoramento do perfil lipídico, realizando pontuações globais e ECC. A realização de eletrocardiograma e ecocardiograma é essencial para 
descartar possíveis alterações arrítmicas e/ou disfunções ventriculares. Com relação à prescrição dos exercícios, os resultados de o eletrocardiograma e o ecocardiograma ajudam na realização de uma estratificação de risco préexercício mas adequada ${ }^{41}$.

Os testes máximos devem ser evitados na PPS devido à fadiga, que pode ser desencadeada devendo a atividade deve ser prescrita com base em testes submáximos. Partindo desse pressuposto o teste de caminhada de seis minutos, apesar de ser de intensidade submáxima surge como uma boa opção ${ }^{41}$.

\section{CONCLUSÃO}

A gestão das doenças neuromusculares, incluindo a SPP, representa um desafio para profissionais de saúde e pacientes. Muitas vezes esse problema pode vir a complicar pela paresia diafragmática e consequentemente problemas cardiorrespiratório, o que pode levar os médicos a seguirem as protocolos invasivos sem considerar os não-invasivos além dos programas de reabilitação. A detecção precoce e reabilitação podem atenuar os transtornos gerados pela SPP e prolonga a qualidade de vida. A gestão do SPP requer atenção específica aos fatores de vulnerabilidade dos sistemas compensatórios, mecanismos de lesão por excesso de uso, oxigenação e uso ineficiente da musculatura enfraquecida. 


\section{É fundamental que exista um equilíbrio entre a}

prescrição de exercício, descanso, atividade, organização do espaço a ser utilizado pelo paciente. Cabe ressaltar que mais estudos e outras análises são necessárias.

\section{REREFÊNCIAS}

1.Bickerstaffe $A$, Beelen A, Nollet F. Change in physical mobility over 10 years in post-polio syndrome. Neuromuscul Disord 2015;25:22530. https://doi.org/10.1016/j.nmd.2014.11.015

2.Esteban J. Polio paralytic: new problems: postpolio syndrome. Rev Esp Salud Publica 2013;87:517-22. https://doi.org/10.4321/S113557272013000500010

3.Trojan DA, Cashman NR. Post-poliomyelitis syndrome. Muscle Nerve 2005;319:6-19. https://doi.org/10.1002/mus.20259

4.Bach JR, Alba AS. Pulmonary dysfunction and sleep disordered breathing as post-polio sequelae: evaluation and management. Orthopedics 1991;14:1329-37. https://doi.org/10.3928/0147-744719911201-07

5.Trojan DA, Arnold DL, Shapiro S, Bar-Or A, Robinson A, Le Cruguel $\mathrm{JP}$, et al. Fatigue in post-poliomyelitis syndrome: association with disease-related, behavioral, and psychosocial factors. PM R 2009;1:442-9. https://doi.org/10.1016/j.pmrj.2009.03.003

6. Fischer JR, Headley J. Post-polio breathing and sleep problems revisited. Post-Polio Health 2004;20:5-7.

7.Garip Y, Eser F, Bodur H, Baskan B, Sivas F, Yilmaz O. Health related quality of life in Turkish polio survivors: impact of post-polio on the health related quality of life in terms of functional status, severity of pain, fatigue, and social, and emotional functioning. Rev Bras Reumatol Engl Ed 2017;57:1-7.

https://doi.org/10.1016/j.rbre.2014.12.006

8.Silva TM, Moreira GA, Quadros AAJ, Pradella-Hallinan M, Tufik S, Oliveira ASB. Analysis of sleep characteristics in post-polio syndrome patients. Arq Neuropsiquiatr 2010;68:535-40.

https://doi.org/10.1590/s0004-282×2010000400011

9.Siegel H, McCutchen C, Dalakas MC, Freeman A, Graham B, Alling $D$, et al. Physiologic events initiating REM sleep in patients with the postpolio syndrome. Neurology 1999;52:516-22.

https://doi.org/10.1212/wnl.52.3.516

10.Laffont I, Julia M, Tiffreau V, Yelnik A, Herisson C, Pelissier J. Aging and sequelae of poliomyelitis. Ann Phys Rehabil Med 2010;53:24-33.

https://doi.org/10.1016/j.rehab.2009.10.002

11.Romigi $A$, Maestri $M$. Circadian fatigue or unrecognized restless legs syndrome? The post-polio syndrome model. Front Neurol 2014;5:115. https://doi.org/10.3389/fneur.2014.00115 
12.Vasconcelos OM, Prokhorenko OA, Salajegheh MK, Kelley KF, Livornese $\mathrm{K}$, Olsen $\mathrm{CH}$, et al. Modafinil for treatment of fatigue in post-polio syndrome: a randomized controlled trial. Neurology 2007;68:1680-6.

https://doi.org/10.1212/01.wnl.0000261912.53959.b4

13. Acler M, Bocci T, Valenti D, Turri M, Priori A, Bertolasi L. Transcranial direct current stimulation (tDCS) for sleep disturbances and fatigue in patients with post-polio syndrome. Restor Neurol Neurosci 2013;31:661-8. https://doi.org/10.3233/RNN-130321

14.Gawne AC, Wells KR, Wilson KS. Cardiac risk factors in polio survivors. Arch Phys Med Rehabil 2003;84:694-6. https://doi.org/10.1016/S0003-9993(02)04836-0

15.Orsini M, Souza JA, Araújo Leite MA, Teixeira S, Sá Ferreira A, Bastos $\mathrm{VH}$, et al. Previous acute polio and post-polio syndrome: recognizing the pathophysiology for the establishment of rehabilitation programs. Neurol Int 2015;7:5452. https://doi.org/10.4081/ni.2015.5452

16. Matyja E. Post-polio syndrome. Part II. Therapeutic management. Neurol Neurochir Pol 2012;46:372-8.

17. Halstead LS, Rossi CD. New problems in old polio patients: results of a survey of 539 polio survivors. Orthopedics 1985;8:845-50.

18. Weinberg J, Borg J, Bevegård S, Sinderby C. Respiratory response to exercise in postpolio patients with severe inspiratory muscle dysfunction. Arch Phys Med Rehabil 1999;80(9):1095-100. https://doi.org/10.1016/s0003-9993(99)90067-9

19.Dolmage TE, Avendano MA, Goldstein RS. Respiratory function during wakefulness and sleep among survivors of respiratory and non-respiratory poliomyelitis. Eur Respir J 1992;5:864-70.

20.Lane DJ, Hazleman B, Nichols PJR. Late onset respiratory failure in patients with previous poliomyelitis. Q J Med 1974;43:551-68.

https://doi.org/10.1093/oxfordjournals.ajmed.a067405

21.Dean E, Ross J, Road JD, Courtenay L, Madill KJ. Pulmonary function in individuals with a history of poliomyelitis. Chest 1991;100:118-23. https://doi.org/10.1378/chest.100.1.118

22. Lira CA, Minozzo FC, Sousa BS, Vancini RL, Andrade MS, Quadros $A A$, et al. Lung function in post-poliomyelitis syndrome: a crosssectional study. J Bras Pneumol 2013;39:455-60. https://doi.org/10.1590/S1806-37132013000400009

23.Ward NS, Hill NS. Pulmonary function testing in neuromuscular disease. Clin Chest Med 2001;22:769-81. https://doi.org/10.1016/S0272-5231(05)70065-4

24.Midgren B. Lung function and clinical outcome in postpolio patients: a prospective cohort study during 11 years. Eur Respir J 1997; 10:146-9.

https://doi.org/10.1183/09031936.97.10010146

25.Boitano LJ. Management of airway clearance in neuromuscular disease. Respir Care 2006;51:913-22.

26. Bach JR, Gonçalves MR, Hon A, Ishikawa Y, De Vito EL, Prado F, et al. Changing trends in the management of end-stage neuromuscular 
respiratory muscle failure: recommendations of an international consensus. Am J Phys Med Rehabil 2013;92:267-77. https://doi.org/10.1097/PHM.0b013e31826edcf1

27. Wolfe LF, Joyce NC, McDonald CM, Benditt JO, Finder J. Management of pulmonary complications in neuromuscular disease. Phys Med Rehabil Clin N Am 2012;23:829-53. https://doi.org/10.1016/j.pmr.2012.08.010

28.Bach JR, Bakshiyev R, Hon A. Noninvasive respiratory management for patients with spinal cord injury and neuromuscular disease. Tanaffos 2012;11:7-11.

29.Orsini M, Lopes AJ, Menezes SL, Oliveira AB, Freitas MR, Nascimento $\mathrm{OJ}$, et al. Current issues in the respiratory care of patients with amyotrophic lateral sclerosis. Arq Neuropsiquiatr 2015;73:873-6. https://doi.org/10.1590/0004-282X20150132

30.van Kralingen KW, Ivanyi B, Keimpema AR, Venmans BJ, Visser M, Postmus PE, et al. Sleep complaints in postpolio syndrome. Arch Phys Med Rehabil 1996;77:609-11. https://doi.org/10.1016/s00039993(96)90304-4

31.Steljes DG, Kryger MH, Kirk BW, Millar TW. Sleep in postpolio syndrome. Chest 1990;98:133-40.

https://doi.org/10.1378/chest.98.1.133

32. Bhat S, Gupta D, Chokroverty S. Sleep disorders in neuromuscular diseases. Neurol Clin 2012; 30:1359-87.

https://doi.org/10.1016/j.ncl.2012.08.010

33.Aboussouan LS. Sleep-disordered breathing in neuromuscular disease. Am J Respir Crit Care Med 2015;191:979-89. https://doi.org/10.1164/rccm.201412-2224CI

34.Kang $\mathrm{JH}$, Lin HC. Comorbidity profile of poliomyelitis survivors in a Chinese population: a population-based study. J Neurol 2011;258:1026-36. https://doi.org/10.1007/s00415-010-5875-y

35.Greenland P, Alpert JS, Beller GA, Benjamin EJ, Budoff MJ, Fayad $\mathrm{ZA}$, et al. $2010 \mathrm{ACCF} / \mathrm{AHA}$ guideline for assessment of cardiovascular risk in asymptomatic adults: a report of the American College of Cardiology Foundation/American Heart Association Task Force on Practice Guidelines. J Am Coll Cardiol 2010;56:e50-103. https://doi.org/10.1016/j.jacc.2010.09.001

36.Stöllberger C, Blazek G, Wegner C, Finsterer J. Neurological comorbidity affects prognosis in left ventricular hypertrabeculation/noncompaction. Heart Lung 2012;41:594-8. https://doi.org/10.1016/j.hrtlng.2012.03.002

37. Nollet F, Beelen A, Sargeant AJ, Visser M, Lankhorst GJ, Jong BA. Submaximal exercise capacity and maximal power output in polio subjects. Arch Phys Med Rehabil 2001;82:1678-85. https://doi.org/10.1053/apmr.2001.27390

38. Kriz JL, Jones DR, Speier JL, Canine JK, Owen RR, Serfass RC. Cardiorespiratory responses to upper extremity aerobic training by postpolio subjects. Arch Phys Med Rehabil 1992;73:49-54. 
39.Dean E, Ross J. Effect of modified aerobic training on movement energetics in polio survivors. Orthopedics 1991;14:1243-6. https://doi.org/10.3928/0147-7447-19911101-13

40.Voorn EL, Gerrits KH, Koopman FS, Nollet F, Beelen A. Determining the anaerobic threshold in postpolio syndrome: comparison with current guidelines for training intensity prescription. Arch Phys Med Rehabil. 2014;95(5):935-40.

https://doi.org/10.1016/j.apmr.2014.01.015

41.Vreede KS, Henriksson J, Borg K, Henriksson M. Gait characteristics and influence of fatigue during the 6-minute walk test in patients with post-polio syndrome. J Rehabil Med 2013;45:924-8. https://doi.org/10.2340/16501977-1209 